\title{
Quantum dot with ferromagnetic leads: a densiti-matrix renormalization group study
}

\author{
C. J. Gazza, M. E. Torio, and J. A. Riera \\ Instituto de Física Rosario, Consejo Nacional de Investigaciones Científicas y Técnicas, \\ Universidad Nacional de Rosario, Rosario, Argentina
}

(Dated: May 12, 2018)

\begin{abstract}
A quantum dot coupled to ferromagnetically polarized one-dimensional leads is studied numerically using the density-matrix renormalization group method. Several real space properties and the local density of states at the dot are computed. It is shown that this local density of states is suppressed by the parallel polarization of the leads. In this case we are able to estimate the length of the Kondo cloud, and to relate its behavior to that suppression. Another important result of our study is that the tunnel magnetoresistance as a function of the quantum dot on-site energy is minimum and negative at the symmetric point.

PACS numbers: 73.63.Kv, 72.15.Qm, 72.25.-b
\end{abstract}

The relentless pursuit of smaller, faster, more efficient electronic devices has led to an increasing interest in mesoscopic and nanoscopic devices behaving as quantum dots (QD), using semiconductor technology ${ }^{\underline{\underline{1}}}$ and nanotubes as components ${ }^{2}$ among other possibilities. Some of these devices allow the exploitation of the electron spin in addition to its charge with various potential applications in the so-called spintronics ${ }^{\frac{3}{3}}$ In particular, a spintronic device connecting a QD to ferromagnetic leads has already been experimentally studied $\underline{4}$

These nanoscopic devices have also been growing in complexity. For example a device consisting of two QDs has been developed to show a nonlocal control of one QD over the other via a RKKY interaction. ${ }^{5}$ Another example is the device where an appropriate arrangement of electrodes defines a section of a nanotube as the $\mathrm{QD} . \underline{6}$ This QD turns out to be then connected to essentially one-dimensional leads. In the first example, a real space description of the magnetic correlations would be important to understand the internal working of this device. In the second example, the effects of finite length of the nanotube sections connected to the QD would have to be considered.

Among many techniques used to study these kinds of problems, which have been developed in the context of the Kondo effect,,$\frac{7}{t}$ the numerical renormalization group (NRG) has provided many important and essentially exact results. However, this technique cannot tackle the increasingly complex devices such as the above mentioned ones. For this reason, we propose in this paper the application of a real space numerical technique, the densitymatrix renormalization group (DMRG) ${ }^{8}$, which has been extensively used to study quasi-one-dimensional strongly correlated electron systems $\stackrel{9}{-}$ This technique is ideally suited to provide detailed real space information, such as spin-spin correlations or electron site occupancies, required to understand nanoscopic devices. In addition, it works on finite size systems. This is not a disadvantage when bulk leads are involved in the device because in most cases one could resort to some kind of extrapolation. On the contrary, it can provide results for finite systems such as the example mentioned before.
In this paper, we will study the simplest device in spintronics, the spin valve. This device consists of a singlelevel QD attached to two noninteracting leads. On these leads, a ferromagnetic polarization is introduced by an applied magnetic field, or in other words, by a rigid displacement of spin-up and spin-down electron bands. The central quantities to study are the conductance when the polarization of the leads are parallel $\left(G_{P}\right)$ and antiparallel $\left(G_{A P}\right)$. The main measure of the performance of the spin valve is the tunnel magnetoresistance (TMR) defined as $T M R=\left(G_{P}-G_{A P}\right) / G_{A P}$. This device has been studied before using different approximations ${ }^{10,11,12}$. These analytical techniques are not exact and in fact they lead to some degree of controversy around central issues such as the behavior of the conductance when the leads have a parallel polarization. Some of these controversial issues have been partially settled using NRG $\underline{\underline{13,14}}$

As in previous studies on this system, the Hamiltonian model for a QD located at the center of the chain is a generalization of the Anderson model defined as

$$
\begin{aligned}
\mathcal{H}= & -t \sum_{i=\leq-2, \sigma}\left(c_{i \sigma}^{\dagger} c_{i+1 \sigma}+\text { H.c. }\right)-h_{L} \sum_{i=\leq-1} S_{i}^{z} \\
& -t \sum_{i=\geq 1, \sigma}\left(c_{i \sigma}^{\dagger} c_{i+1 \sigma}+H . c .\right)-h_{R} \sum_{i=\geq 1} S_{i}^{z} \\
& -t^{\prime} \sum_{\sigma}\left(c_{-1 \sigma}^{\dagger} c_{0 \sigma}+c_{0 \sigma}^{\dagger} c_{1 \sigma}+\text { H.c. }\right) \\
& +\epsilon n_{0}+U n_{0, \uparrow} n_{0, \downarrow}
\end{aligned}
$$

where conventional notation was used. We adopt $t$ as the scale of energy. The magnetic field on the leads can be set in two configurations, $h_{L}=h_{R}=h$, which corresponds to a parallel $(\mathrm{P})$ polarization of the leads, and $h_{L}=-h_{R}=h$ which corresponds to the antiparallel (AP) one. In the following, $h>0$ favors a positive polarization. Notice that the leads are described by a real-space tight-binding model which is in principle more realistic than the ones implicit in the NRG treatment.

Model equation(11) was studied by the DMRG on $L=63,79$ and 95 chains with open boundary conditions. Most of the results shown below were obtained for two sets of parameters: $U=1, t^{\prime}=0.4$ and $U=4$, 


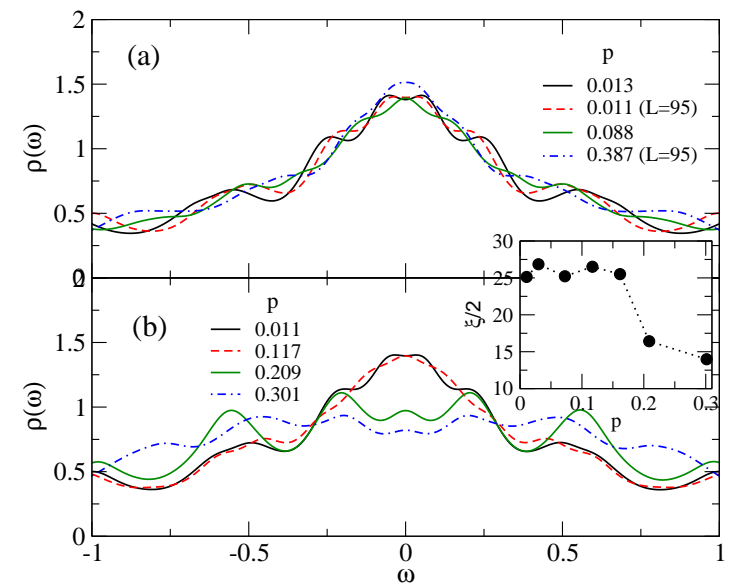

FIG. 1: (Color online) The LDOS for $U=1, t^{\prime}=0.4$, and $\epsilon=-0.5$, for various values of the polarization $p$, (a) AP magnetization for the $L=79$ chain, and (b) $\mathrm{P}, L=95$. The inset shows the length of the "compensation cloud" (see text) for the $\mathrm{P}$ case, $L=95$.

$t^{\prime}=0.8$. For both sets of parameters the effective Kondo coupling at the symmetric point is $J=4 t^{\prime 2} / U=0.64$, although strictly speaking this relationship is only valid for $U>>t^{\prime}$. There are, however, many important differences between both sets of parameters as we will show below.

The main quantity that we have studied is the local density of states (LDOS), $\rho(\omega)$, at the QD. In the first place, from this quantity it is possible to evaluate the conductance in the linear response regime. In the second place, recent advances in scanning tunneling microscopy (STM) have made it possible to directly measure this quantity. Since in our DMRG calculation the ground state vector is measured when the two added sites are at the center of the chain (symmetrical configuration), the QD then is one of these two sites which are exactly treated. Then, we adopt the approximation of applying the creation and annihilation operators at the QD on the ground state vector and then determine $\rho(\omega)$ following the well-known continued fraction formalism. A more accurate approach would be, after the application of each of those creation and annihilation operators, to run additional sweeps for an enlarged density matrix $\frac{15}{n}$ In any case, the truncation of the Hilbert space is the essential source of error in the DMRG, and to estimate the precision of our approach we have compared results for various numbers of retained states $M$, from $M=500$ to 1000 . We would also like to stress the fact that the conductance in linear response is related to the LDOS near $\omega=0$ where the approximation is more precise $\underline{16}$ In addition, we have computed standard properties such as the electron occupancy of each site, $<n_{i, \sigma}>(\sigma=\uparrow, \downarrow)$ and spin-spin correlations from the $\mathrm{QD}, S(j)=<S_{0}^{z} S_{j}^{z}>-<S_{0}^{z}><S_{j}^{z}>$.

All the results presented correspond to the half-filled system. For the AP case, we work in the $S^{z}=1 / 2$ sector. For the parallel polarization, we work in the $S^{z}$ subspace

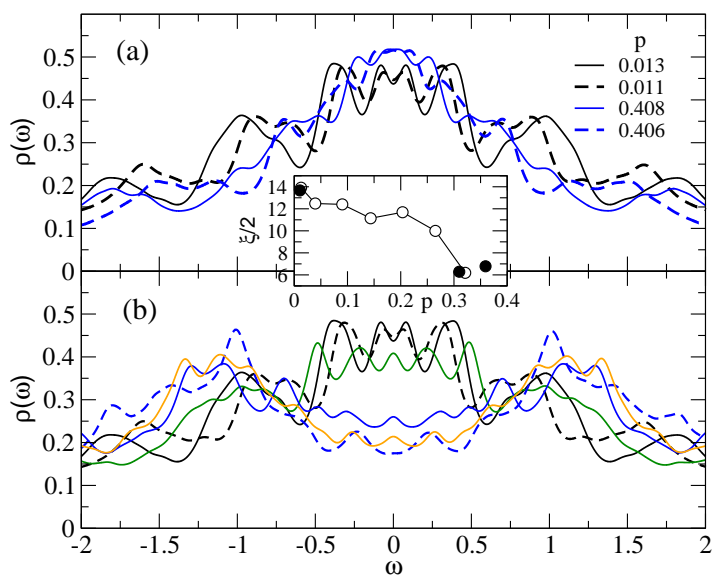

FIG. 2: (Color online) The LDOS for $U=4, t^{\prime}=0.8$, and $\epsilon=-2.0$, for various values of the polarization $p$, (a) antiparallel, $L=79$ (solid lines), $L=95$ (dashed lines); (b) parallel configuration, from top to bottom at $\omega=0$ : $L=79, p=0.013,0.203,0.321,0.426$ (solid lines); $L=95$, $p=0.011,0.399$ (dashed lines). The inset shows the length of the "compensation cloud" (see text) for the P case, $L=79$ (open circles), $L=95$ (filled circles).

which minimizes the total energy, and hence it depends on $h$. The polarization of each lead $p_{\alpha}(\alpha=L, R)$ is defined as $p_{\alpha}=\left(n_{\alpha \uparrow}-n_{\alpha \downarrow}\right) /\left(n_{\alpha \uparrow}+n_{\alpha \downarrow}\right)$. Then, the polarization $p$ of the $\mathrm{P}(\mathrm{AP})$ configuration is $\left(p_{L}+p_{R}\right) / 2$ $\left[\left(p_{L}-p_{R}\right) / 2\right]$. For small clusters, and specially for the $U=1$ case, it is difficult to get $p_{L}=p_{R}$, i.e. the same numbers of solitons on each lead. In these cases the AP case shows spurious suppression of the LDOS 17

In Fig. 11we show the LDOS at the QD, $U=1, t^{\prime}=0.4$, at the symmetric point $\epsilon=-U / 2$, for several values of the polarization $p$. In this figure and all the following similar ones, we adopted a Lorentzian broadening of the peaks of $\delta=0.1$ In the first place, for $p=0.013$, corresponding to $h=0$, the LDOS is the one expected in the Kondo problem. The small splitting of the Kondo peak is a finite size effect. For the case of an AP configuration (Fig. प(a)), a small value of $p=0.088$ leads to a slight modification of the LDOS, that virtually remains unmodified by further increasing the polarization of the leads, at least in the range examined $(p \leq 0.3)$. The largest value considered, $p=0.3$, is obtained for $h=2.2(L=95)$. On the other hand, for the $\mathrm{P}$ configuration (Fig. 1(b)) it can be seen as a suppression of the Kondo peak for the polarization $p \geq 0.2$. For small values of $p$ the peak at $\omega=0$ remains roughly unmodified. As we will show below, this different dependence of the LDOS with $p$ for the AP and $\mathrm{P}$ arrangements leads to the expected behavior of a spin valve. The inset in Fig. 1 shows, for the $\mathrm{P}$ magnetization, the length of the "compensation cloud"18, a possible measure of the Kondo cloud, defined in such a way that the sum of the correlations $S(j)(-\xi / 2 \leq j \leq \xi / 2, j \neq 0)$ is equal to $0.9 S(0), 17$ The reduction of $\xi$ with $p$, due to the better screening of the minority spin at the QD by the majority spins on the leads, is consistent with the suppression of 


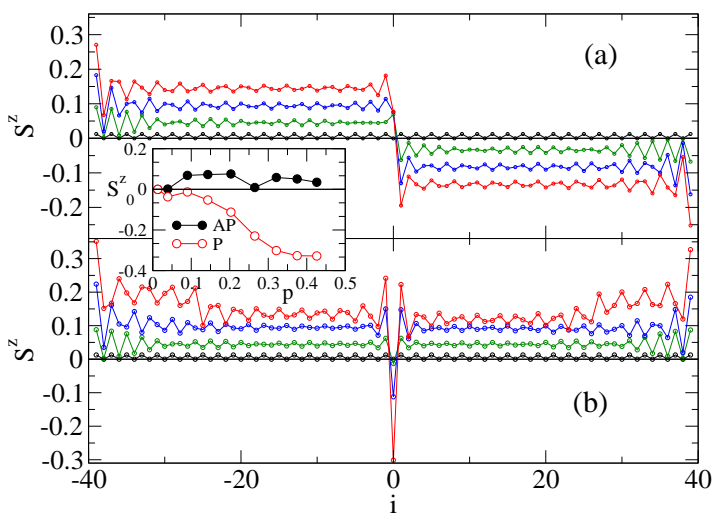

FIG. 3: (Color online) $S_{i}^{z}$ for the $L=79$ chain, $U=4, t^{\prime}=$ $0.8, \epsilon=-2.0$, for $p \approx 0.01,0.1,0.2$ and 0.3 , from bottom to top on the left lead; (a) antiparallel, (b) parallel configuration. The inset shows $S^{z}$ at the QD as a function of the polarization

the Kondo resonance observed in the LDOS.

Qualitatively the same behavior can be observed for the case of the parameters $U=4, t^{\prime}=0.8$, at the symmetric point $\epsilon=-2.0$. In this case, the Kondo resonance has a smaller weight as compared with the previous set of parameters. In Fig. 22 a), which shows the LDOS around $\omega=0$, it can be seen again that for the AP case the LDOS is not much changed as $p$ is increased. In contrast, for the $\mathrm{P}$ case, the Kondo peak is strongly suppressed in the presence of polarization. This suppression is considerably stronger than for the previous set of parameters, $U=1$, $t^{\prime}=0.4$. We have also examined the size dependence of these results by computing the LDOS on the $L=95$ chain. Although the position of the peaks, as expected, moves to lower frequencies (in absolute value), the overall behavior with $p$ is similar to that for $L=79$. As in the previous case, the length of the "compensation cloud" (shown in the inset in Fig. 2) for the $\mathrm{P}$ polarization decreases with increasing $p$ in agreement with the suppression of the Kondo resonance. It is interesting to note that, consistently with this behavior, the value of $\left\langle\left(S_{0}^{z}\right)^{2}\right\rangle$ also decreases from $\sim 0.18$ for $p \sim 0$ to $\sim 0.10$ for $p \sim 0.4$. Notice that in the full Kondo regime $\left\langle\left(S_{0}^{z}\right)^{2}\right\rangle \sim 0.25$.

The mean value of $S_{i}^{z}$ is shown in Fig. 3 for $U=4$, $t^{\prime}=0.8$, and $\epsilon=-2.0$, for the AP and $\mathrm{P}$ polarizations in panels (a) and (b), respectively. The inhomogeneous way in which the sites are polarized, due to the open boundary conditions of our clusters, is clearly visible. We have also observed this behavior for the same parameters on the 95-site cluster, suggesting that it is not a mere finite size effect. The different behavior of the AP and $\mathrm{P}$ configurations can be traced to the behavior of the mean value of the spin at the QD. The apparently different behavior of $S_{0}^{z}=S_{Q D}^{z}$ as a function of the polarization is shown in the inset. For the AP arrangement $S_{0}^{z}$ is almost not affected by $p$, while for the $\mathrm{P}$ arrangement $S_{0}^{z}$ goes to the minimum possible value by increasing $p$. This behavior of $S_{0}^{z}$ in the $\mathrm{P}$ case is well known and it has been shown that a magnetic field applied to the QD could in fact reduce the suppression of the LDOS

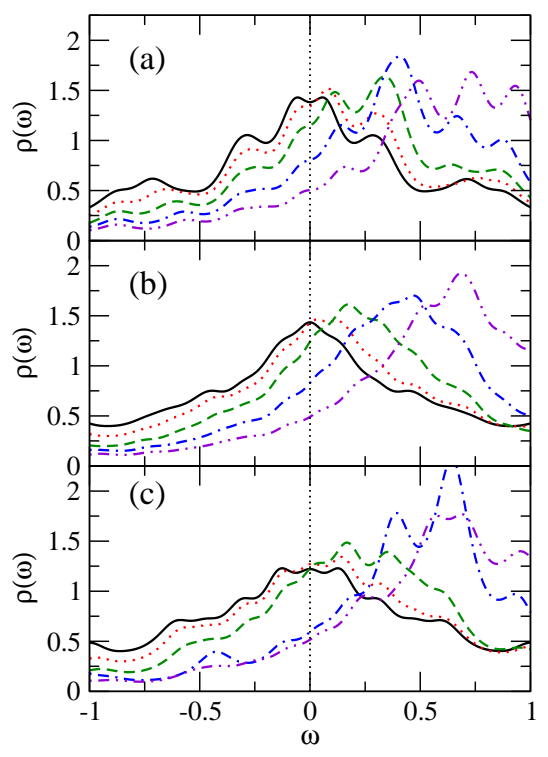

FIG. 4: (Color online) The LDOS for the $L=79$ chain: $U=$ $1, t^{\prime}=0.4$, and $\epsilon=-0.5$ (solid line), -0.25 (dotted line), 0.0 (dashed line), 0.25 (dotted-dashed line), 0.5 (dotted-dotteddashed line) for (a) $p=0.0$, (b) $p=0.30$, AP configuration, and (c) $p=0.30$, parallel configuration.

\section{discussed above 12.13}

We now study the most important issue in this kind of systems, i.e., the behavior of the conductance and hence of the TMR as a function of the applied voltage. To understand this behavior we show in Fig. 4 the LDOS on the $L=79$ chain for the parameters $U=1, t^{\prime}=0.4$, lead polarization $p=0.3$, and various values of $\epsilon$. In Fig. (4) (a) we show for comparison the unpolarized case. The relevant quantity is the LDOS at $\omega=0$ which is proportional to the conductance, $G=\left(2 \pi t^{\prime 2} / t\right) \rho(\omega=0)$, in units of $e^{2} / h$. $G$ as a function of $\epsilon$ has the typical form of a dome as it can be seen in Fig. [5. (a). However, $\rho(\omega=0)$ for a finite cluster is an artifact of the Lorentzian broadening $\delta$ of the peaks adopted in our calculations. Although $\rho(\omega=0)$ could be computed in this way ${ }^{19}$, we prefer to discuss the results of Fig. 目 at a qualitative level. The LDOS for $p=0.3$ in the AP polarization is shown Fig. प(b). Its $\omega$ dependence for various values of $\epsilon$ is very similar to the one for the unpolarized case. On the other hand, for the $\mathrm{P}$ configuration, the LDOS, depicted in Fig. 田(c), shows at $\omega=0$ a nonmonotonic behavior as a function of the gate voltage. This nonmonotonic behavior, when translated to the conductance, is crucial to understand the $\epsilon$ dependence of the TMR.

Our most relevant results are shown in Fig. 5 To compute the conductance we use the Friedel sum rule $\mathrm{z}^{\underline{\underline{7}}}$, $G_{\sigma}=\sin ^{2} \pi n_{\sigma}$, where $n_{\sigma}$ is the occupancy at the QD of an electron with spin $\sigma$. The Friedel sum rule is valid for arbitrary polarization in the $\mathrm{P}$ case, except for negligible higher order corrections $\underline{13}$. The total conductance is $G=G_{\uparrow}+G_{\downarrow}$.

Figure [5(a) shows the conductance for the unpolarized case, $\mathrm{AP}$ and $\mathrm{P}$ polarizations, for $U=1, t^{\prime}=0.4$, and 

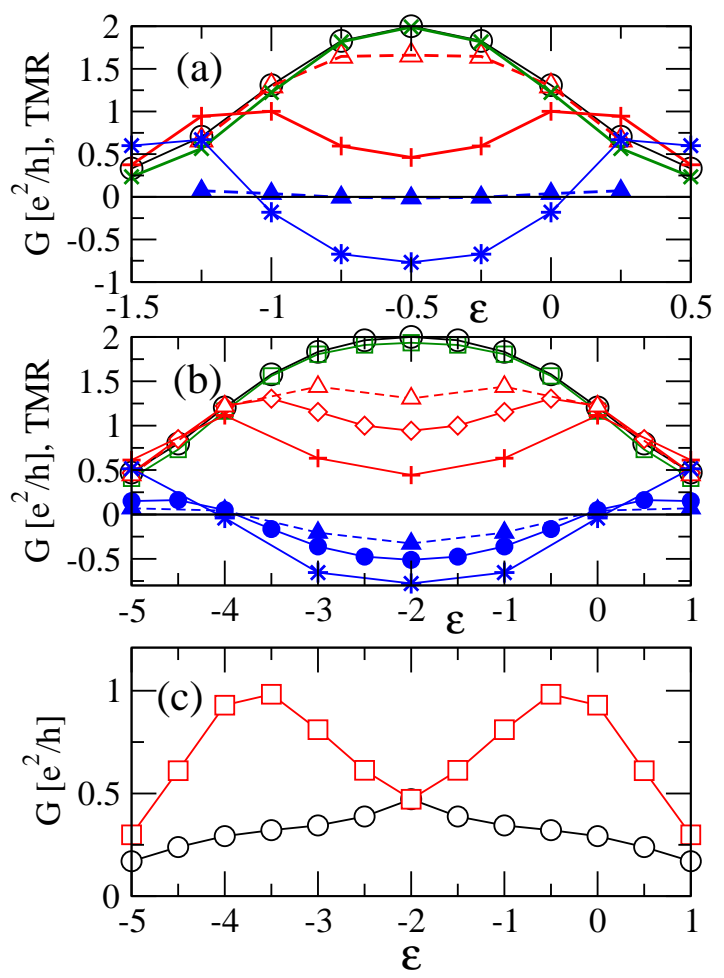

FIG. 5: (Color online) (a) Conductance as a function of the gate voltage for $U=1, t^{\prime}=0.4, L=79$, and $p=0$ (open circles). Results for $L=63, p=0.30$ : P configuration (open triangles), TMR (filled triangles), and $L=95, p=0.35 \mathrm{AP}$ (crosses), P (plusses), and TMR (stars). (b) Same as (a) for $U=4, t^{\prime}=0.8$. Symbols for $L=63$ and $L=95$ same as (a). Results for $L=79$ : AP (squares), P (diamonds), and TMR (filled circles). The values of the polarization are $p=0.31$ $(L=63), p=0.32(L=79)$, and $p=0.36(L=95)$. (c) Upspin (circles) and down-spin (squares) contributions to $G_{P}$ for the parameters of (b), $L=79$.

Fig. 5(b) for $U=4, t^{\prime}=0.8$. The conductance for the AP configuration is virtually indistinguishable from the unpolarized one for $L=79$. On the other hand, $G_{P}$ has a minimum at the symmetric point, then increases as $\epsilon$ moves apart from that point, and finally it merges with the curve for the unpolarized case, decreasing for large values of the gate voltage. Then, in the most important region for application purposes, i.e. near the symmetric point, the TMR is negative with a minimum precisely at this point. This result is in contradiction with the one reported in Ref. 14, showing a positive value at the symmetric point. This difference may be traced to the model we adopted for the leads, which implies loosing the particle-hole symmetry for the $\mathrm{P}$ case, which is present in Ref. 14. ${ }^{20}$ Notice that for the case of Fig. 5](b), there is a clear trend as the chain size is increased, that is, a deepening of $G_{P}$ around the symmetric point, with a subsequent decrease in the TMR. Notice that the lead polarization is slightly different in the three clusters. This trend is not that clear for the case of Fig. [5(a), since here the smaller value of $U$ requires larger lattice sizes.

Finally, the up- and down-spin contributions to $G_{P}$ are shown in Fig. 5) (c) for the couplings of Fig. 5(b). At the symmetric point $G_{\uparrow}=G_{\downarrow}$. As $\epsilon$ is moved away from the symmetric point, the conductance of the minority spin becomes dominating, reaching perfect conductance, at $\epsilon \sim-U$ and $\epsilon \sim 0$, and it accounts for the increase of the total conductance.

In summary, we have shown the possibility of applying the DMRG technique to the problem of transport through quantum dots. For the particular problem of a quantum dot coupled to polarized leads we have shown that the LDOS at the QD is suppressed by the $\mathrm{P}$ polarization of the leads. In addition, we have shown that the TMR as a function of the quantum dot on-site energy is minimum and negative at the symmetric point, a result which is at variance with those reported in previous studies. This is a central issue in these kinds of devices. In addition to this, we have shown that this technique allowed us to estimate the length of the Kondo cloud, and to relate its behavior with the suppression of the Kondo peak, correlated in turn with the conductance. This emphasizes the fact that real space properties, which are accessible by the DMRG, and are very difficult to obtain by alternative techniques, are very important to understand the behavior of these devices.

\section{Acknowledgments}

We acknowledge useful discussions with A. E. Feiguin, S. Maekawa, J. Martinek, and G. B. Martins. This work was supported by grant No. PICT 03-12409 (ANPCYT).
1 D. Goldhaber-Gordon et al, Nature 391, 156 (1998)

2 S. J. Tans, A. R. M. Verschueren, C. Dekker, Nature 393, 49 (1998); K. Tsukagoshi et al, Nature 401, 572 (1999).

3 S. A. Wolf et al, Science 294, 1488 (2001). S. Maekawa and T. Shinjo, Spin Dependent Transport in Magnetic Nanostructures. (Taylor \& Francis, London, 2002).

4 A. N. Pasupathy et al, Science 306, 86 (2004); J. Nygaard, et al, cond-mat/0410467

5 N. J. Craig, et al, Science 304, 565 (2004).

${ }_{7}$ M. J. Biercuk, et al, Nano Letters 5, 1267 (2005).

7 A. J. Hewson, The Kondo problem to heavy fermions,
(Cambridge University Press, Cambridge, UK, 1993).

8 S. R. White, Phys. Rev. Lett. 69, 2863 (1992); Phys. Rev. B 48, 10345 (1993).

9 I. Peschel, et al, Density-Matrix Renormalization, Lecture Notes in Physics, vol. 528 (Springer, Berlin, 1999).

10 J. Martinek et al, Phys. Rev. Lett. 91, 127203 (2003).

11 B. Dong et al, J. Phys. Condens. Matter 15, 8435 (2003).

12 I. Weynman et al, Phys. Rev. B 72, 113301 (2005).

13 J. Martinek et al, Phys. Rev. Lett. 91, 247202 (2003); Y. Utsumi et al, cond-mat/0501172 (2005).

14 M. S. Choi, D. Sánchez, and R. López, Phys. Rev. Lett. 
92, 056601 (2004); R. López and D. Sánchez, Phys. Rev. Lett. 90, 116602 (2003).

15 K. A. Hallberg, Phys. Rev. B 52, 9827 (1995); E. Jeckelmann, Phys. Rev. B 66, 045114 (2002).

16 Our calculation starts to be less precise as we move away from $\omega=0$ since then higher excited states are involved.

17 M. E. Torio, C. J. Gazza and J. A. Riera, in preparation.

18 J. E. Gubernatis, J. E. Hirsch, and D. J. Scalapino, Phys.
Rev. B 35, 8478 (1987).

19 In this case $\delta$ should decrease by increasing $L$. See e.g., H. Benthien, F. Gebhard, E. Jeckelmann, Phys. Rev. Lett. 92, 256401 (2004).

20 This has been recently shown by J. Martinek et al, Phys. Rev. B 72, 121302(R) (2005). 